\title{
Acetabulum fracture sequelae
}

\author{
Raffaele Pascarella, Simone Cerbasi, Simone Finzi \\ Section of Orthopaedic \& Trauma Surgery, Ospedali Riuniti, Ancona, Italy
}

\begin{abstract}
SUMMARY
Outcomes in patients with acetabulum fractures are related to many factors, both intrinsic and extrinsic. Intrinsic factors includes age, type of fracture, and dislocation of the femoral head. Extrinsics factors include the timing of dislocation reduction, treatment indication, timing of surgical treatment, accuracy of the fracture reduction, and joint wear over time. We will evaluate the various factors by analyzing the predictability of a failure or a complication. This work aims to highlight the critical issues related to complex fractures such as those of the acetabulum and the resulting outcomes. We will focus mainly on extrinsic factors that can be acted on to achieve better results.
\end{abstract}

Key words: acetabulum fracture, surgical treatment, outcome

Received: December 5, 2019

Accepted: January 6, 2020

\section{Correspondence}

Simone Cerbasi

Section of Orthopaedic \& Trauma Surgery, Ospedali Riuniti, via Conca 71, 60030 Ancona, Italy

E-mail: simone.cerbasi@ libero.it

Conflict of interest

The Authors declare no conflict of interest

How to cite this article: Pascarella R, Cerbasi S, Finzi S. Acetabulum fracture sequelae. Lo Scalpello 2020;34:42-6. https://doi. org/10.36149/0390-5276-006

(C) Ortopedici Traumatologi Ospedalieri d'Italia (O.T.O.D.i.) 2020

\section{(c) (1) $(9)$}

This is an open access article distributed in accordance with the CC-BY-NC-ND (Creative Commons Attribution-NonCommercial-NoDerivatives 4.0 International) license. The article can be used by giving appropriate credit and mentioning the license, but only for non-commercial purposes and only in the original version. For further information: https://creativecommons.org/licenses/by-nc-nd/4.0/deed.en

\section{Introduction}

Acetabulum fractures are very complex lesions, often associated with major trauma, which frequently involve other systems and other segments of the skeleton. In the last 25 years, with the aging of the population and, in particular, in patients over the age of 60 , there has been an increase in incidence of 2.4 times of acetabular fractures The mechanism of injury in this age group differs substantially to that of young adults. Low energy falls represent $50 \%$ of acetabular fractures in patients over 60 years of age ${ }^{1-4}$. The classification of these fractures is fundamental to decide the most correct treatment and if there is a surgical indication (and eventually the type of access, reduction and type of osteosynthesis). Patients with a fracture of the acetabulum diagnosed with standard radiography of the pelvis should always undergo CT with two-dimensional and three-dimensional reconstructions. No decision can be made if the patient is not subjected to these investigations. CT indeed shows the fracture in all its extension, if both acetabular columns are involved, if there are impacted articular fragments or intra-articular fragments, if there is a dislocation or subluxation of the femoral head, or if there are osteochondral lesions of the femoral head. Three-dimensional reconstructions are very important for deciding on surgical planning and which approach to use ${ }^{5}$. The fracture must be classified according to Judet's classification ${ }^{6}$. This classification is useful for several reasons. Firstly, it allows the clinician to perfectly frame the type of lesion. Furthermore, each type of fracture is related to different surgical approaches, different results and different complications.

The dislocation of the femoral head, which is often associated with these fractures, causes frequent complications and must be immediately reduced. After reduction, a CT scan should always be performed to assess the presence of intra-articular fragments. If the $\mathrm{CT}$ was already performed during the initial diagnostic tests, it must be repeated after femoral head reduction.

\section{Outcome}

Some outcomes are related to predictable but not modifiable factors such as the 
type of fracture or the necrosis of the femoral head. Other outcomes are instead due to a wrong surgical indication, to an error in the timing of the reduction of the femoral epiphysis dislocation, to suboptimal fracture reduction or to an error during the surgical treatment. These complications are predictable and therefore modifiable ${ }^{2,7-10}$.

Factors influencing outcomes can be divided into intrinsic and extrinsic.

Among the intrinsic factors there are:

- patient's age;

- type of fracture;

- femoral epiphysis dislocation.

Extrinsic factors include:

- dislocation reduction timing;

- fracture reduction timing;

- surgical indication;

- fracture reduction;

- surgical technique;

- joint wear.

\section{Intrinsic factors}

\section{Patient age}

The age of the patient affects the characteristics of the fracture, the possibility of obtaining optimal reduction and the stability of the means of synthesis. In patients over 60 years a deterioration in bone quality is observed, especially in women. For this reason elderly patients often show poly-fragmented fractures, with acetabular roof or posterior wall impacted fragments resulting in severe joint damage ${ }^{11}$. Moreover, in these patients there may be difficulties in reducing the fracture as the gripping elements of the pincers do not have a mechanical seal, the screws can pull-out, and the bone can fragment in an attempt of reduction. The screws may not have a good seal and a loss of fracture reduction may be observed in a short distance from the surgery.

\section{Type of fracture}

The most complex fractures, involving both acetabular columns, determine the greatest difficulties of reduction, especially if they are surgically treated over 10 days after the trauma. This happens because the fibrous callus, very precocious and abundant in cortico-cancellous bone, prevent the mobilization of fragments and thus their reduction ${ }^{7}$.

Fractures that present an impacted fragment, involving the roof or the posterior wall of the acetabulum, have poorer prognosis, both for the reductive difficulties and for the joint damage that will lead to post-traumatic arthrosis ${ }^{12-14}$.

Sometimes acetabular fracture is associated with femoral head impaction or cartilaginous damage. This situation almost always leads to early articular degeneration and a poor clinical result even in the presence of an anatomical fracture reduction ${ }^{15}$.

\section{Femoral epiphysis dislocation}

Outcomes mainly related to this lesion are due to femoral head necrosis. This complication is closely related to the timing of the reduction. If the dislocation is reduced within the first 6-8 hours after the trauma the percentage of necrosis is very low, less than $5 \%$. When the reduction occurs at $>24$ hours the percentage of necrosis can reach $40 \%{ }^{7-10}$.

\section{Extrinsic factors}

\section{Timing}

Timing is a fundamental element to achieve good results ${ }^{7}$. The reduction of the femoral head dislocation, as mentioned, must take place immediately, in the emergency room (Fig. 1), except in cases where the general conditions of the patient are compromised.

The dislocation is not reduced by inserting a transfemoral traction and connecting weights, but specific reductive maneuvers are required with the patient in narcosis. It may happen that a closed reduction is impossible. This occurs in the presence of large intra-articular fragments or of a part of capsule that is interposed preventing the closed reduction. In these cases an urgency intervention must be performed if an experienced surgeon is available otherwise surgery has to be procrastinated to the following day. To leave a hip dislocated will lead, in a high percentage of cases, to necrosis of the femoral epiphysis and therefore to a negative outcome ${ }^{7-10}$.

The timing in surgical treatment of the fracture is another key element to improve outcomes. These fractures, especially the more complex ones, must be treated surgically within 10 days of the injury. The fragments will still be mobile and a single surgical access may be required. After 14 days the fibrous callus will make the reduction, and thus achieving a good result, much more difficult ${ }^{7}$.

\section{Surgical indication}

There are precise rules on surgical indications. An acetabulum fracture must be treated surgically when the displacement of the fragments is greater than 2-3 mm, in the presence of intra-articular fragments or impacted fragments, in the presence of dislocation or subluxation of the femoral epiphysis ${ }^{7,8}$. In patients over 60 years of age, in the presence of complex fractures, non-operative treatment and subsequent total hip arthroplasty can be considered, but a total hip replacement surgery in an untreated acetabular fracture can be very complicated, due to the anatomical subversion and the possible loss of bone, and it may be necessary to use a revision implant, with all the resulting consequences. Acetabulum fractures should be treated surgically with internal reduction and osteosynthesis even in people over 60, as in most cases we will get good results. If the patient will develop a secondary coxarthrosis it will be possible to use a first implant prosthesis, with long survivals and a lower rate of complications $3,4,11,16$. 


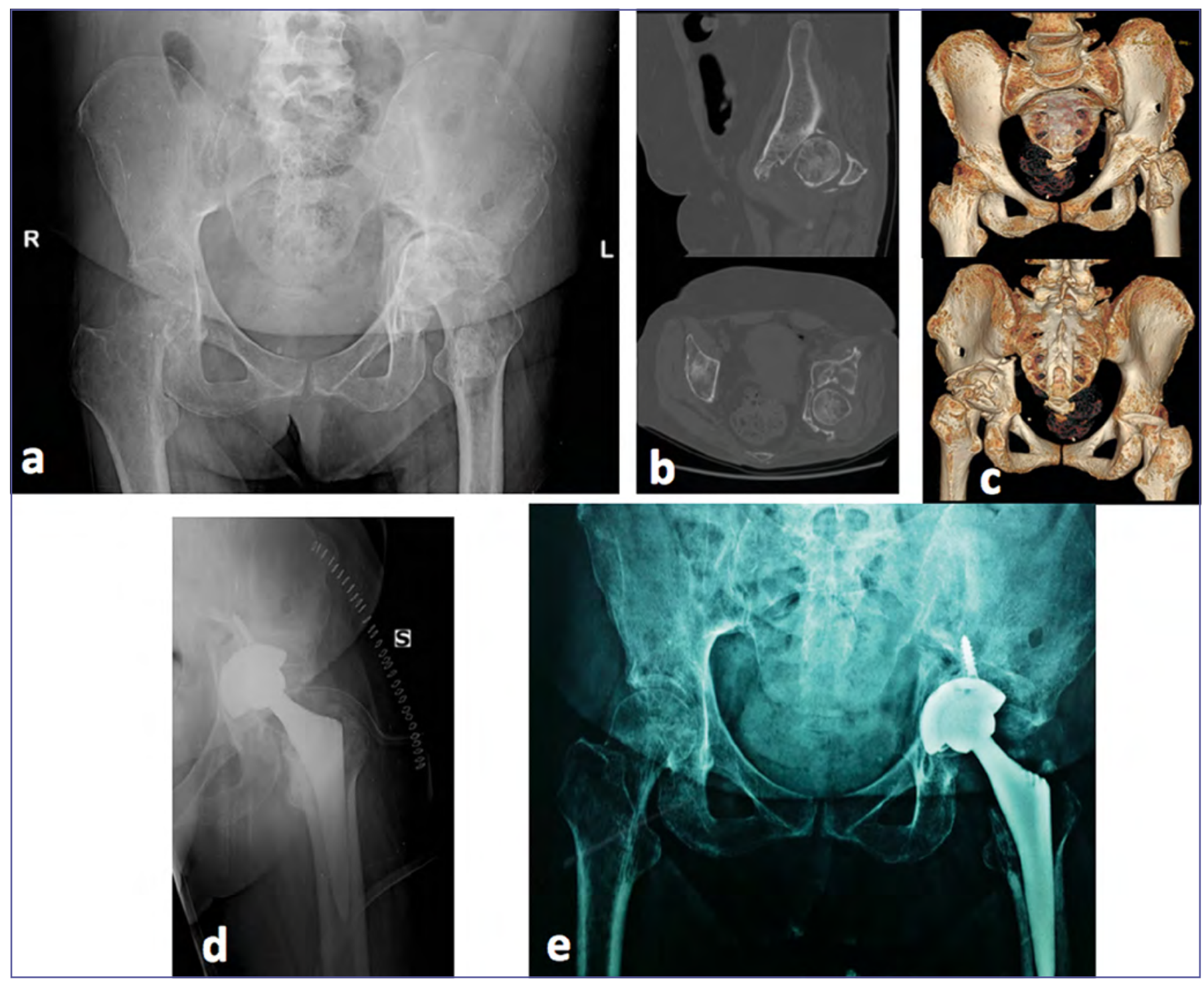

Figure 1. Example of sequeale. Inveterate acetabular fracture and dislocation of the femoral head. Poor treatment. Patient developed post-traumatic osteoarthritis (a-c). This complication is predictable and therefore modifiable. Immediate (d) and one-year (e) post-operative performed with THA.

\section{Fracture reduction}

Acetabulum fractures, like all joint fractures, must be reduced anatomically. Matta ${ }^{7}$ considers a fracture well reduced when the residual decomposition is 0 or less than $1 \mathrm{~mm}$. Over $3 \mathrm{~mm}$ is considered an unsatisfactory reduction, and therefore not acceptable. An anatomic reduction is not always possible when we are in the presence of complex fractures, in patients over the age of 60 or when more than 14 days have elapsed since the trauma. For these reasons, the traumatized patient should be moved to the operating room as soon as possible, as soon as the general conditions allow it. In addition, patients with acetabulum fractures should only be treated in specialized centers, exceeding 20 to 30 cases per year. Operating 5 cases a year is meaningless and will inevitably lead to bad results.

\section{Surgical technique}

Acetabular fractures require a great deal of surgical experience ${ }^{2,6,7,9}$. It is not a surgery that can be improvised (Fig. 2). The surgeon must possess, in addition to experience, a radiolucent surgical table, which allows obtaining the different projections of Judet intra-operatively, and the reduction pincers dedicated to this surgery. The use of the image intensifier throughout the surgery will allow the correct positioning of the clamps, an assessment of the obtained reduction, control of the direction and length of the screws. 


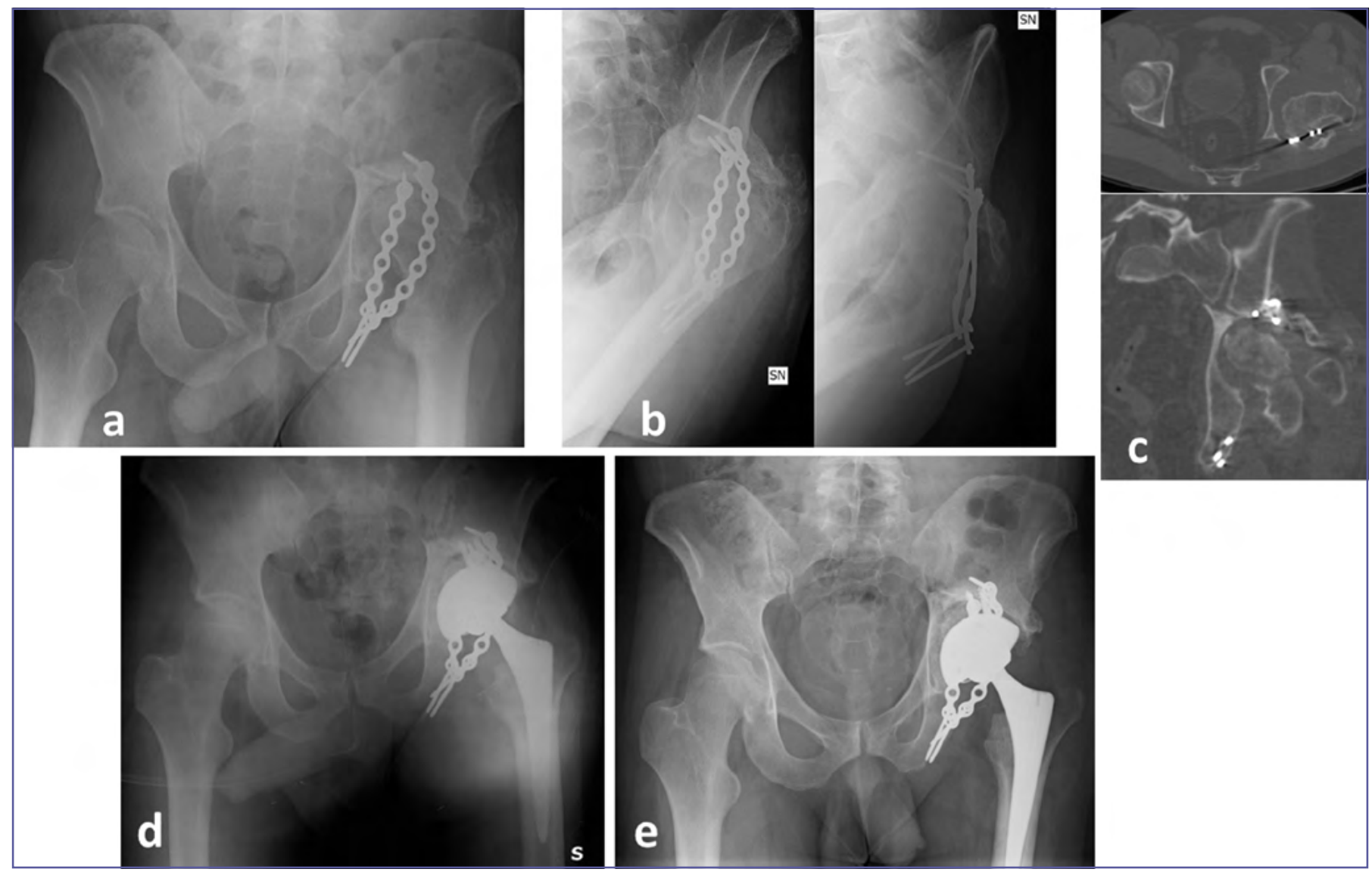

Figure 2. Example of a incongruent reduction with dislocated femoral head leading to advanced changes in the hip joint (a-c). Immediate (d) and one-year (e) post-operative performed with THA.

\section{Joint wear}

This last factor can be considered both intrinsic, because we cannot oppose the passage of time, and extrinsic because the reduction obtained will change the fate of the joint with an early arthrosis or with long survivals. It is, however, natural that after 10-15 years from the traumatic event a fractured joint may undergo slow wear and therefore present post-traumatic arthrosis ${ }^{1,2,17-19}$.

\section{Discussion and conclusions}

Acetabulum fractures are very complex lesions that require indepth diagnostics, a precise classification and careful surgical

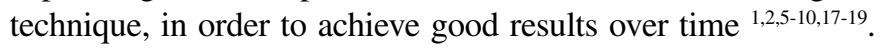
Correctly classifying an acetabulum fracture is of fundamental importance both to plan the subsequent treatment and to consider the possible correlated outcomes and therefore to make the patient aware of his pathology and possible complications ${ }^{5}$. Fractures that present impacted fragments of the acetabular roof often have an early post-traumatic osteoarthritis either due to the difficulty in reducing these fragments, or to a subsequent chondrolysis or an acetabular necrosis ${ }^{14,15}$. Another negative prognostic factor is observed in fractures in which the femoral head is incarcerated in the acetabular roof with an epiphyseal osteo-cartilaginous damage. In these cases, very often, the patient needs a hip replacement within one year of the trauma. The other very important intrinsic factor is the posterior dislocation of the femoral head. Any damage to the epiphyseal vasculature is established at the time of the trauma but it worsens until the dislocation is reduced ${ }^{7-10}$. For this reason, the reduction must take place in the first hours after the trauma. It is of no use to apply traction to the patient unless the dislocation is first reduced. Traction can be useful only if the hip, after reduction, is unstable and tend to dislocate again. The outcomes resulting from these factors cannot be modified but are very important from a prognostic point of view and the patient must be informed.

Extrinsic factors, on the other hand, are related to our decisions and actions and we have to minimize their influence in order to achieve a good result. To obtain an anatomical reduction interventions should be performed as soon as possible, within 7-10 days of the trauma ${ }^{7}$. It is not advisable to operate in the first 2-3 days as we may have major bleeding and a potential instability of the patient. Pre-operative planning is essential. We 
must carefully study the CT results and consider the fracture and the different fragments that compose it, in order to choose the best surgical approach, especially in the more complex lesions such as the transverse and the two columns. The reduction must be anatomical, even if it is not possible in all cases, to minimize the risk of a possible post-traumatic osteoarthritis. It is highlighted that good reduction is the one that foresees a residual displacement of less than $1 \mathrm{~mm}^{7-10}$. To have good results in these fractures a long cultural and surgical preparation is absolutely necessary ${ }^{2,6,7,9}$. These patients must be centralized in hospitals that treat at least 20 to 30 cases each year ${ }^{7}$. Lack of experience and a lower number of cases frequently result in sub-optimal results. It is not sufficient to know how to apply plaques to a pelvis if we are unable to reduce the fracture.

In conclusion, some post-traumatic outcomes are inevitable, related to the type of fracture or to the dislocation of the femoral head. Other outcomes, on the other hand, are related to failure to diagnose, inaccurate planning, unacceptable fracture reduction or poor surgical technique. Regarding these last factors we must act to increasingly improve the clinical and radiographic results of patients admitted to our hospitals.

\section{References}

1 Laird A, Keating JF. Acetabular fractures: a 16 year prospective epidemiological study. J Bone Joint Surg Br 2005;87:969-73.

2 Giannoudis PV, Grotz MR, Papakostidis C, et al. Operative treatment of displaced fractures of the acetabulum. A meta-analysis. J Bone Joint Surg 2005;87:2-9.

3 Butterwick D, Papp S, Gofton W, et al. Acetabular fractures in the elderly: evaluation and management. J Bone Joint Surg Am 2015;97:758-68.

4 Daurka JS, Pastides PS, Lewis A, et al. Acetabular fractures in patients aged $>55$ years: a systematic review of the literature. Bone Joint J 2014;96:157-63.

5 Zinghi GF, Pascarella R. Acetabolo - Lesioni Traumatiche Dall'urgenza al trattamento programmato. Bologna: Timeo 2012.

6 Letournel E. Acetabulum fractures: classification and management. Clin Orthop Relat Res 1980;151:81-106.
7 Matta JM. Fractures of the acetabulum: accuracy of reduction and clinical results in patients managed operatively within 3 weeks after the injury. J Bone Joint Surg Am 1996; 78:1632-45.

8 Moed BR, Yu PH, Gruson KI. Functional outcomes of acetabular fractures. J Bone Joint Surg Am 2003;85:1879-83.

9 Meena UK, Tripathy SK, Sen RK, et al. Predictors of post-operative outcome for acetabular fractures. Orthop Traumatol Surg Res 2013;99:929-35.

10 Pascarella R, Cerbasi S, Politano R, et al. Surgical results and factors influencing outcome in patients with posterior wall acetabular fractures. Injury 2017;48:1819-24.

11 Manson T, Schmidt AH. Acetabular fractures in the elderly: a critical analysis review. JBJS Rev 2016;4.

12 Saterbak AM, Marsh JL, Nepola JV, et al. Clinical failure after posterior wall acetabular fractures: the influence of initial fracture patterns. J Orthop Trauma 2000;14:230-7.

13 Moed BR, Willson Carr SE, et al. Results of operative treatment of fractures of the posterior wall of the acetabulum. J Bone Joint Surg Am 2002;84:752-8.

14 Martins e Souza P, Giordano V, Goldsztajn F, et al. Marginal impaction in posterior wall fractures of the acetabulum. AJR Am J Roentgenol 2015;204:470-4.

15 Rommens PM, Ingelfinger P, Nowak TE, et al. Traumatic damage to the cartilage influences outcome of anatomically reduced acetabular fractures: a medium-term retrospective analysis. Injury 2011;42:1043-8.

16 De Bellis UG, Legnani C, et al. Acute total hip replacement for acetabular fractures. A systematic review of the literature. Injury 2014;45:356-61.

17 Magu NK, Gogna P, Singh A, et al. Long term results after surgical management of posterior wall acetabular fractures. J Orthop Traumatol 2014;15:173-9.

18 Wright R, Barrett K, Christie MJ, et al. Acetabular fractures: longterm follow-up of open reduction and internal fixation. J Orthop Trauma 1994;8:397-403.

19 Mitsionis GI, Lykissas MG, Motsis E, et al. Surgical management of posterior hip dislocations associated with posterior wall acetabular fracture: a study with a minimum follow-up of 15 years. J Orthop Trauma 2012;26:460-5. 